\title{
O PAPEL DO COMPORTAMENTO NA OCORRÊNCIA DE CASCATAS TRÓFICAS
}

\author{
Rafael Dettogni Guariento ${ }^{1,2 *}$ \\ ${ }^{1}$ Laboratório de Limnologia, Depto. de Ecologia, Instituto de Biologia, Universidade Federal do Rio de Janeiro (UFRJ). Ilha do Fundão. CEP: 21941 - \\ 590, Caixa Postal: 68020. Rio de Janeiro, Brasil. \\ ${ }^{2}$ Programa de Pós-Graduação em Ecologia, Inst. de Biologia, UFRJ. Ilha do Fundão. CEP: 21941-590, Caixa Postal: 68020. Rio de Janeiro, Brasil. \\ *E-mail: dettogni@biologia.ufrj.br
}

\section{RESUMO}

A cascata trófica é reconhecidamente um importante mecanismo na regulação em cadeias tróficas embora um grande debate exista a respeito dos mecanismos finais que determinam a ocorrência deste processo. Cascatas tróficas podem emergir a partir de efeitos diretos entre populações, no qual predadores consomem suas presas e, portanto, diminuindo a abundância de presas que influenciam níveis tróficos inferiores. Porém, as cascatas tróficas também podem emergir a partir de efeitos comportamentais indiretos, nos quais as presas alteram seu comportamento de forrageamento em resposta ao risco de predação. Estas mudanças comportamentais afetam o esforço de forrageamento da presa, diminuindo seu impacto sobre os recursos. Neste trabalho é apresentada uma síntese das evidências empíricas que suportam o papel das interações indiretas mediadas por mudanças comportamentais, como mecanismos causais da expressão de cascatas tróficas em diferentes ecossistemas. Observa-se que respostas comportamentais das presas em função da presença de predadores são comuns em uma grande variedade de ecossistemas afetando de forma significativa padrões observados no nível de comunidades e de ecossistemas. É também observado que a contribuição relativa dos efeitos mediados pela densidade ou por mudanças comportamentais pode variar dependendo do contexto biótico ou abiótico em que estas interações estão inseridas. Conclui-se como base no grande número de evidências levantadas que a incorporação da perspectiva comportamental na teoria de cadeias tróficas se faz necessária na tentativa de elucidar os mecanismos que controlam as cascatas tróficas bem como o funcionamento e a estrutura de comunidades ecológicas.

Palavras-chave: Cascata trófica, forrageamento adaptativo, efeitos indiretos mediados pela densidade, efeitos indiretos mediados pelo comportamento, interação entre predadores e presas.

\begin{abstract}
THE ROLE OF THE BEHAVIOR ON THE INCIDENCE OF TROPHIC CASCADES. Trophic cascades are well accepted mechanisms of top-down regulation in food webs, although its ultimate causes are still in debate. Trophic cascades can emerge through direct effects between populations, where predator consume their prey, decreasing the abundance of preys that affect lower trophic levels. However, trophic cascades can also emerge through indirect behaviorally mediated effects, so that the prey alter its foraging regime in response to predation risk. Such behavioral shifts affect prey foraging effort, reducing its impact on resources. This study presents a synthesis of empirical evidences that support behaviorally mediated indirect interactions as ultimate causes of trophic cascades in a wide range of ecosystems. It was observed that behavioral prey responses in the presence of predators are common in a wide array of ecosystems, strongly affecting patterns at the community and ecosystems levels. It's also noticed that the relative contribution of density or behaviorally mediated effects depends on the biotic or abiotic environmental context. As a conclusion, based on the empiral and theorectical data gathered until now, the use of the behavioral perspective on the food web theory is necessary as an attempt to elucidate the mechanisms that rule trophic cascades and the functioning and structure of ecological communities
\end{abstract}

Keywords: Trophic cascades, adaptive foraging, density mediated indirect interactions, behavioral mediated indirect interactions, predator-prey interactions. 


\section{INTRODUÇÃO}

Tradicionalmente, os modelos que buscavam compreender a dinâmica das interações entre predadores e presas baseavam-se em características e forças de interação 'fixas' entre espécies (Murdoch \& Oaten 1975, Rosenzweig \& MacArthur 1963, Hassell 1978), substituindo as espécies como unidades básicas da comunidade, pelo seu conjunto de características físicas e funcionais. Neste contexto, assume-se que o universo decaracterísticas que determinamahabilidade dos predadores em capturar suas presas como, por exemplo: tamanho do corpo, velocidade, aparato bucal, bem como as características das próprias presas se modificariam de forma lenta quando comparadas às dinâmicas populacionais que determinam os padrões observados no nível de comunidades ou ecossistemas. Em outras palavras, por mais que estas características sejam modificadas pela seleção natural imposta pela interação entre as espécies, este processo não atuaria de forma significativa no tempo ecológico de interesse. Esta perspectiva foca-se no efeito letal que os predadores exercem sobre suas presas e que as interações são propriedades intrínsecas de duas espécies que interagem e, portanto governadas pelas suas respectivas densidades. No contexto de cadeias tróficas, o efeito indireto propagado pela mudança nas densidades de duas espécies que interagem diretamente, é conhecido como "interação indireta mediada pela densidade" (DMII - derivado do inglês density mediated indirect interaction - Abrams 1995). No entanto, um problema fundamental a esta perspectiva surge se uma espécie reage na presença de uma segunda espécie alterando seu fenótipo (ex. comportamento, morfologia, fisiologia), esta mudança pode modificar seu efeito per capita em outras espécies, mesmo na ausência de mudanças na densidade. Esta nova perspectiva baseia-se no efeito que o predador possui em 'assustar' a sua presa. A presa (indivíduo) trata o risco de predação como um custo na sua atividade de forrageamento e responde através de mudanças em seu fenótipo. De fato, a capacidade do predador de assustar suas presas é uma propriedade que está mais relacionada às presas que aos predadores. Se a presa exibe um comportamento conspícuo na presença de determinado predador, este pode ser considerado neste caso como 'assustador'. Como conseqüência, esta plasticidade de características dá origem a uma outra forma de efeito indireto que pode ser transmitido ao longo das cadeias tróficas, conhecida como "interação indireta mediada por características" (TMII - derivado do inglês trait mediated indirect interaction - Abrams 1995). Assim, as interações entre predadores e presas podem ser entendidas a partir de duas perspectivas complementares, embora, de certa forma divergentes, cada uma focando em diferentes aspectos ou mecanismos que governam estas interações. Fenótipos influenciados pelo ambiente foram considerados menos importantes por biólogos evolutivos devido a aparente falta de fundamentação genética. No entanto, a noção moderna da plasticidade fenotípica assume que esta plasticidade possui bases genéticas podendo em muitas circunstâncias, ser considerada como adaptativa (Dudley \& Schmitt 1996).

Considerando as interações entre predadores e presas bem como a busca por alimentos ou parceiros, é evidente que em organismos terrestres superiores a sinalização não se dá apenas pelo contato visual, mas também, através dos sentidos da audição e do olfato. Entretanto, em ecossistemas aquáticos estes tipos de comunicação são menos prováveis e a comunicação através de substâncias químicas (Cairomônios - Figura 3) que são liberadas pelos organismos é considerada como um dos principais mecanismos de sinalização mediando uma série de interações (Brönmark \& Ander-Hanson 2000, Ruther et al. 2002). O reconhecimento de substâncias químicas liberadas por outros organismos foi identificado para um grande número de taxa e algumas revisões sobre este tipo de comunicação existem para diversas sub-áreas como busca de parceiros (Olsen et al. 2000); navegação (Stabell 1992); busca de alimento (Stabell 1992); reconhecimento de predadores (Kats \& Dill 1998).

Apesar das evidências teóricas e experimentais sugerirem que TMIIs sejam importantes na estruturação de comunidades (Abrams 1992, 1995, Werner 1992, Peacor \& Werner 2000), DMIIs são consideradas com muita maior freqüência, tanto em modelos matemáticos ou teóricos como em explicações mecanicistas de evidências empíricas a respeito da dinâmica de comunidades ecológicas. No entanto, TMIIs, devido a respostas comportamentais, são provavelmente bastante comuns na natureza (Werner 1992). Considerando que as taxas de 
crescimento e o risco de predação estão positivamente relacionados à atividade de forrageamento, a maioria dos organismos estão sujeitos a um trade-off em que a alocação de energia em determinado aspecto levará ao comprometimento do outro, um processo que pode ocasionar TMIIs. Por exemplo, a atividade de forrageamento deve diminuir com o aumento do risco de predação ou com o aumento da quantidade de recursos. Portanto, ambos, predadores e competidores podem induzir mudanças comportamentais que levem a TMIIs. Somada a isto, a plasticidade fenotípica foi extensivamente documentada para diversos taxa (Tollrian \& Harvell 1999), evidenciando que os componentes necessários para TMIIs são plausíveis e que TMIIs podem ser quantitativamente importantes na maioria das teias tróficas.

O objetivo deste trabalho é fazer uma síntese das evidências empíricas e teóricas que suportem as interações indiretas mediadas por mudanças comportamentais induzidas pelos predadores em suas presas, como mecanismos causais da expressão de cascatas tróficas em diferentes ecossistemas. Finalmente, comparar os efeitos relativos de DMIIs e TMIIs em diferentes contextos ecológicos e sugerir as direções futuras neste campo de estudo.

\section{CASCATAS TRÓFICAS MEDIADAS POR TMIIS}

A idéia de que as comunidades ecológicas podem ser visualizadas como cadeias lineares compreendendo produtores, herbívoros e carnívoros interagindo entre si, levou à predição de um importante fenômeno em ecologia de comunidades conhecido como 'cascata trófica' (Paine 1980). A cascata trófica pode ser definida como os efeitos indiretos que o predador possui em níveis tróficos inferiores atuando diretamente em níveis tróficos intermediários. No entanto, a visão clássica da ocorrência de cascata trófica é baseada na redução numérica da população de herbívoros provocada por carnívoros através de seu consumo direto. Os poucos herbívoros remanescentes exerceriam um efeito fraco nos produtores, indiretamente os carnívoros exercem um efeito positivo dos produtores (DMII). À medida que a presa exibe um comportamento furtivo em relação ao predador, é provável que o impacto da presa nos produtores também diminua (Abrams 1995), seja reduzindo sua atividade ou alternando de habitat, levando a ocorrência de cascata trófica por TMII (Figura 1).
Vários estudos conduzidos em uma variedade de ecossistemas, focando diferentes organismos, conseguiram isolar o efeito comportamental do predador em suas presas e identificaram como estes efeitos se propagavam nas cadeias tróficas. Trabalhando em rios, McIntosh \& Townsend (1996) observaram que o impacto da herbivoria de efemerópteros (Deleatidium) na biomassa de algas bentônicas é reduzido na presença de trutas (Salmo trutta). Na presença da truta, os efemerópteros despendiam um maior tempo na procura de refúgios, o que diminuía sua atividade de forrageamento. Peckarsky \& McIntosh (1998) também estudando o impacto de efemerópteros (Baetis bicaudatus) na biomassa de algas bentônicas em rios, observaram que sinais de alarme produzidos por trutas (Salvelinus fontinalis) e a presença de plecópteros (Megarcys signata) favoreciam a biomassa algal através da redução da atividade dos herbívoros ou sua mudança de habitat, respectivamente. Turner (1997) e Bernot \& Turner (2001), através de estudos em poças naturais e artificiais, observaram que o impacto de gastrópodes (Physa integra) na biomassa perifítica é menor na presença de peixes (Lepomis gibbosus e Orconectes rusticus) ou apenas na presença dos sinais químicos deixados pelos predadores na água. Em geral, os gastrópodes limitavam sua área de forrageamento a regiões cobertas que representavam um refúgio aos predadores. $\mathrm{O}$ aumento do uso de refúgios também foi observado por Nyström \& Abjörnsson (2000) estudando o comportamento de indivíduos de Rana temporaria na presença de sinais químicos liberados por trutas Oncorhynchus mykiss. O aumento na utilização dos refúgios levou a diminuição da herbivoria sobre o perifíton, aumentando a biomassa perifítica através de efeitos indiretos mediados pelo comportamento do herbívoro - TMII.

As evidências de TMIIs não se limitam a ambientes aquáticos. Messina (1981) observou que a presença de formigas do gênero Formica reduzia o impacto de besouros (Trirhabda) em uma espécie de erva (Solidago altissima) muito comum em pradarias norte americanas. As formigas impediam a defoliação das plantas mesmo não afetando a densidade de besouros nos caules destas. Berckerman et al. (1997), também trabalhando em pradarias, observaram que na presença de aranhas (Pisaurina mira) gafanhotos da espécie Melanoplus femirrubrum alternavam de 


\section{Redução na Atividade de Forrageamento}

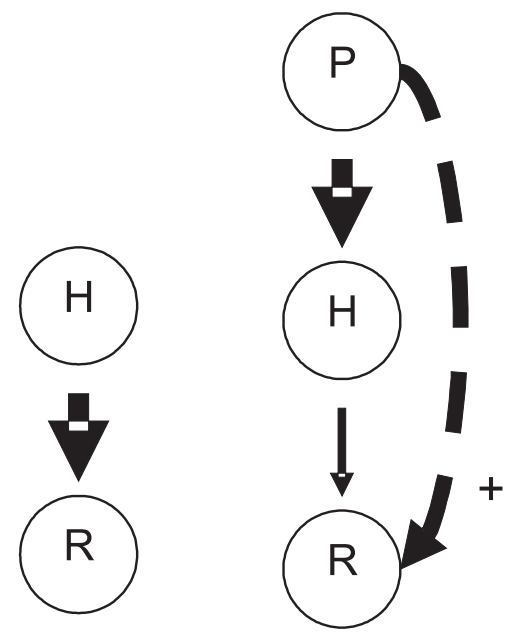

Alternância de Habitat

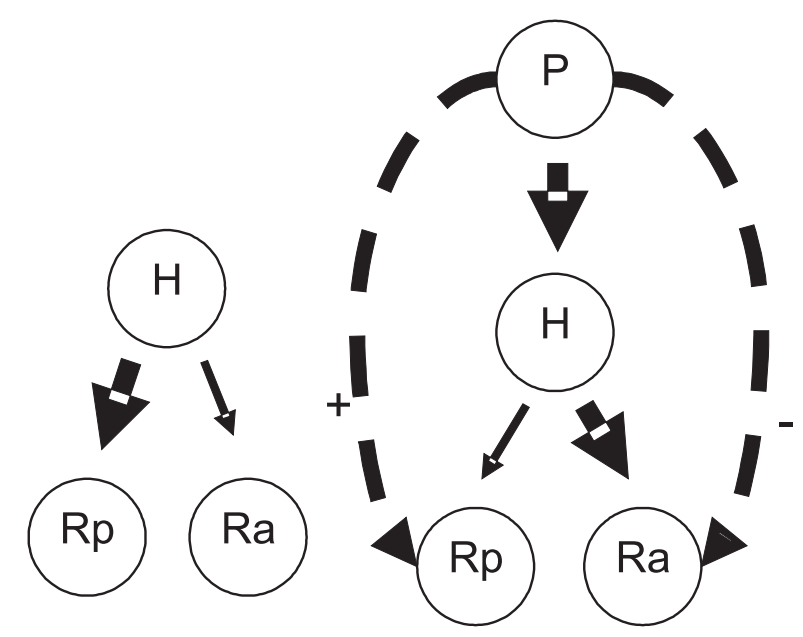

Figura 1. Topologia de cadeias tróficas e os efeitos indiretos resultantes da mudança comportamental dos herbívoros (H) em função dos predadores (P). Linhas contínuas representam interações diretas e linhas tracejadas representam interações indiretas. A espessura das linhas indica a força da interação. A figura esquematiza as possíveis interações na ausência e na presença de predadores. Redução na taxa de herbivoria reduz o impacto nos recursos (R), levando a um efeito positivo indireto do predador sobre os recursos. A alternância de habitat do herbívoro leva à diminuição do impacto sobre o recurso preferencial (Rp) e aumento do uso de recursos alternativos (Ra), com efeitos positivos e negativos dos predadores sobre os recursos. Modificado de Schmitz et al. (2004).

habitat, diminuindo o risco de mortalidade, reduzindo seu impacto na biomassa de gramíneas. Casos de TMIIs também foram detectados para florestas tropicais (Gastreich 1999), costões rochosos (Trussell et al. 2002) e lagos (Carpenter et al. 1987, Diehl \& Eklov 1995), abrangendo a mesma amplitude de ecossistemas examinados em revisões recentes sobre fatores que determinam a força de cascatas tróficas (Borer et al.2005). A ocorrência de cascata trófica por TMII pode ter efeitos tanto positivos quanto negativos sobre recursos (Figura 1). Schmitz (1998), construindo experimentalmente cadeias tróficas com gramínea, ervas, gafanhotos e aranhas, mostrou que na presença de aranhas os gafanhotos alternavam sua dieta para uma maior proporção de ervas. Vale ressaltar que as aranhas tinham sua porção bucal colada com um tipo de cimento o que prevenia o consumo das presas. Assim, a biomassa de gramíneas era 1,55 a 1,66 vezes maior na presença de aranhas com um efeito líquido negativo na biomassa de ervas. Wissinger \& McGrady (1993) quantificaram as interações entre duas espécies de libélulas, dentre os quais um predador de topo (Tramea) que pode se alimentar da outra (Erithemis), que por sua vez se alimenta de tricópteros. Excluindo o efeito letal do predador de topo, através da remoção de lobos distais do aparato bucal, eles observaram que a presença de Tramea reduzia em $61 \%$ o número de tricópteros consumidos por Erithemis. Indivíduos de Tramea intactos reduziam a densidade de Erithemis em 25\%. Assumindo que o efeito de DMII de Tramea nos tricópteras fosse então da magnitude de $25 \%$, os autores sugeriram que naquelas condições experimentais os efeitos de TMIIs eram mais que o dobro dos de DMIIs.

\section{COMPARANDO OS EFEITOS DE TMII E DMII}

Quão importantes são as TMIIs comparados as DMIIs nas cadeias tróficas? Efeitos mediados pela densidade ou pelo comportamento podem variar de magnitude à medida que a densidade de predadores varia. Apenas alguns poucos predadores poderiam desencadear fortes respostas comportamentais em toda população de presas (Feener 2000), enquanto que estes poucos predadores teriam um impacto direto pequeno em suas presas. Entretanto, quando os predadores estão em grande densidade eles podem ter grandes efeitos na densidade das presas e pouco efeito adicional na resposta comportamental ou qualquer outra mudança fenotípica. Torna-se extremamente complicado avaliar empiricamente estes impactos, pois na presença do predador tanto TMII como 
DMII atuariam. Outro fator importante é o fato de os efeitos da densidade e de plasticidade fenotípica não necessariamente possuírem o mesmo sinal (positivo ou negativo), o que torna mais difícil avaliar suas importâncias relativas.

Poucos estudos buscaram separar os efeitos líquidos do predador nos componentes comportamentais e de densidade (Wissinger \& McGrady 1993, Huang \& Sih 1991). Um dos mais bem elaborados trabalhos experimentais que buscou particionar a contribuição de TMII e DMII para o efeito líquido de predadores foi o de Peacor \& Werner (2001). Utilizando ninfas de Odonatas do gênero Anax (predador) e duas classes de tamanho de girinos do gênero Rana, estes autores conduziram um experimento fatorial cruzando quatro níveis de resposta comportamental (quatro diferentes densidades de Anax presas em gaiolas) com três níveis de densidade (remoção manual dos indivíduos) em girinos de pequeno porte. Assim, eles puderam avaliar independentemente os efeitos comportamentais e de densidade nos indivíduos de Rana de menor tamanho e os impactos na biomassa de produtores (perifiton) e nos outros indivíduos de Rana de maior tamanho (competidores). Os resultados foram posteriormente utilizados para separar a contribuição dos dois mecanismos no efeito de indivíduos de Anax livres, capazes de consumir suas presas e induzir efeitos comportamentais. O efeito líquido do predador através da presa tanto nos recursos como no competidor foi bastante claro. O efeito creditado às DMIIs foi de apenas 14 a $24 \%$, e os restantes 76 a $86 \%$ foram atribuídos às TMIIs e a interação entre DMIIs e TMIIs. Os autores concluíram que os efeitos de TMII foram preponderantes nas condições experimentais analisadas.

Werner \& Peacor (2003) sugerem que ao longo de um período de tempo finito, a redução (comportamental) na atividade de forrageamento da presa na presença de um predador é imediata, afetando toda a população e atuando em todo o período de tempo. Assim, o efeito cumulativo do predador mediado pelo comportamento ao longo do tempo de vida da coorte pode ser bastante significativo. Por outro lado, reduções na densidade ocorrem de forma gradual ao longo do tempo e os efeitos são transmitidos apenas a uma porção dos indivíduos e não a toda população. Desta forma, em casos onde os predadores exercem um grande impacto no esforço de forrageamento das presas, espera-se um forte efeito mediado pelo comportamento e grandes modificações nas interações com outras espécies da teia trófica. É possível que muitos dos efeitos tradicionalmente atribuídos ao consumo de presas pelos predadores sejam causados, pelo menos parcialmente, pelos seus efeitos nos sobreviventes da população (Werner \& Peacor 2003). O trabalho de Trussell et al. (2002) é particularmente interessante uma vez que contradiz exemplos clássicos de efeitos em cascata que antes eram atribuídos a DMII. Lubchenco (1978) trabalhando em costões rochosos mostrou que camarões da espécie Carcinus maenas tinham um forte efeito positivo indireto na diversidade e abundância de algas fucóides por consumirem gastrópodes do gênero Littorina, que por sua vez tinham as algas fucóides como um importante item em sua dieta. No entanto, Trussell et al. (2002) utilizando os mesmos organismos, impediram experimentalmente que $C$. maenas consumissem os gastrópodes, colocando-os em tubos perfurados em plots contendo Littorina e algas. Os sinais de predação liberados pelos camarões inibiram a presença de gastrópodes em manchas próximas, observando-se efeitos similares ao obtido por Lubchenco (1978), apenas através de TMII.

Através de modelos matemáticos, Luttbeg et al. (2003) examinaram como o comportamento da presa em determinados contextos ambientais e como determinados protocolos experimentais afetavam a intensidade dos efeitos de TMII e DMII. Estes autores mostraram que a importância relativa de TMIIs comparadas a DMIIs dependia do nível de recursos, sendo TMIIs mais intensas quando os recursos são abundantes. Estes resultados estão de acordo com predições baseadas da ecologia comportamental, onde à medida que a quantidade de recursos diminui, os organismos assumem um maior risco, estando sujeitos a maiores taxas de predação (Ludwig \& Rowe 1990, Werner \& Anholt 1993). Anholt \& Werner (1995) observaram que a taxa de predação em larvas de anuros (Rana) diminui com o aumento da quantidade de recursos. Peacor \& Werner (1997), também utilizando larvas de anuros, mostraram que o aumento na competição, provavelmente levando a uma diminuição da quantidade de recursos, aumentava as taxas de predação. Assim, os resultados obtidos por Luttbeg et al. (2003) devem ser válidos para sistemas em que o esforço de forrageamento das presas diminui com o aumento da disponibilidade de recursos. 
Portanto, experimentos conduzidos em condições de baixa disponibilidade de recursos podem levar à conclusão de que TMII não são importantes, ou ao contrário, que todo efeito indireto transmitido pelo predador através de suas presas são devidos a TMIIs em condições de alta disponibilidade de recursos.

Luttbeg et al. (2003) também mostraram que, dependendo do estágio do ciclo de vida da presa, a contribuição relativa de DMII e TMII deve variar. Se a mudança no comportamento não depende apenas da presença de uma outra espécie, mas também de características da história de vida da presa, a força de TMIIs também deve depender da história de vida da presa. Em estágios iniciais, o comportamento das presas deve ser pouco sensível ao risco de predação (Luttberg et al. 2003). Em estágios iniciais do ciclo de vida, os organismos devem estar próximos aos limites de inanição, e, portanto o acúmulo de energia é necessário. Como resultado, predadores exerceriam pouco efeito nas taxas de forrageamento das presas. Schmitz et al. (1997) trabalhando com ninfas de gafanhoto em estádios iniciais e intermediários encontraram que tanto TMII como DMII ocorriam ao analisar os efeitos de aranhas em gafanhotos e seus recursos, embora apenas TMIIs fossem observadas para ninfas em estádio avançado (Beckerman et al. 1997).

\section{EFEITOS DA IDENTIDADE DO PREDADOR NA NATUREZA DA INTERAÇÃO}

Para prever a ocorrência de TMIIs nas cadeias tróficas, é preciso considerar as características específicas de cada predador as quais induzem as mudanças comportamentais em sua presa (Bernot \& Turner 2001). Devido ao fato de predadores apresentarem diferentes modos de caça e distribuição, eles acarretam diferentes respostas no comportamento das presas (McIntosh \& Peckrsky 1999, Turner et al. 1999). Efemerópteros da espécie Baetis bicaudatus evitam o contato com trutas (Salvelinus fontenalis) escondendo-se sobre rochas, mas a mesma espécie evita contato com plecópteros (Megarcys signata) de outra forma, deixando-se levar pela correnteza para outras regiões (Peckarsky \& McIntosh 1998). $S$. fontenalis e $M$. signata são predadores ativos, mas enquanto a truta forrageia em áreas abertas, o plecóptero busca seu alimento no fundo de rios e riachos. A resposta diferencial do efemeróptero aos diferentes predadores altera sua distribuição espacial e influencia significativamente a distribuição da biomassa de algas bentônicas. Outro exemplo é fornecido por Bernot \& Turner (2001) estudando os efeitos de Lepomis gibbosus (sunfish) e Orconectes rusticus (crustáceo da família cambaridae) em gastrópodes do gênero Physa. L. gibbosus é um predador ativo que atua em áreas abertas na coluna d'água, enquanto $O$. rusticus aguarda sua presa em locais específicos e adota uma estratégia de emboscada. Quando ameaçados por L. gibbosus, os gastrópodes se refugiam em locais cobertos, já quando ameaçados por $O$. rusticus, eles migram para regiões próximas à superfície d'água (Bernot \& Turner 2001). Consequentemente, O. rusticus provoca uma redução na biomassa perifítica em áreas abertas e um aumento na biomassa perifítica em áreas cobertas, sendo o oposto observado na presença de L. gibbosus .

Schmitz \& Suttle (2001) observaram que uma espécie de gafanhoto (Melanoplus. femurrubrum) era predada por três diferentes espécies de aranhas em estudos conduzidos em pradarias. As aranhas da espécie Pisaurina mira são predadores que ficam na copa dos campos e adotam a estratégia senta-eespera. Já indivíduos de Phidilipus rimator, caçam ativamente sua presa por toda a copa dos campos. Rabidosa rabida é um outro predador que adota a estratégia senta-e-persegue nos extratos inferiores dos campos, aguardando sua presa em locais específicos. Os gafanhotos alternam sua dieta de gramíneas para ervas na presença das comparativamente sedentárias $P$. mira e $R$. rabida, mas não mudam seu comportamento na presença de P. rimator (Schmitz \& Suttle 2001). $P$. mira tem efeito indireto positivo sobre as gramíneas e negativo sobre as ervas, porém como não foram observadas diferenças na densidade de gafanhotos a cascata trófica foi inteiramente atribuída a TMII (Schmitz \& Suttle 2001). O tratamento contendo $R$. rabida reduziu significativamente a densidade de gafanhotos, o que levou a um efeito indireto positivo sobre as gramíneas e negativo sobre ervas - DMII e TMII. Finalmente, $P$. rimator causou uma redução significativa na densidade de gafanhotos levando a um efeito positivo em gramíneas e ervas somente por DMII.

O fato das presas apresentarem respostas divergentes na presença de diferentes predadores ou diferentes estratégias de predação sugere que as presas 
percebem as ameaças específicas de cada predador. Predadores que estão continuamente presentes num mesmo habitat fornecem sinais permanentes às presas, tornando a alternância de habitat ou a redução na atividade de forrageamento favorável às presas. Por outro lado, predadores ativos com ampla utilização do habitat possivelmente fornecem sinais por todo o habitat, forçando a presa a pesar o custo energético de sobrevivência com a resposta contínua contra a probabilidade de encontrar e ser capturado pelo predador. Presas que enfrentam predadores muito ativos possivelmente são as que menos apresentam respostas comportamentais, considerando o custo associado a evitar continuamente o predador (Bouskila 2001). Assim, a resposta da presa, em ultima análise, seria determinada pelos custos e benefícios da resposta ao predador com particular modo de caça e utilização de habitat. Este fato levou Schmitz et al. (2004) a proporem a hipótese de que cascatas tróficas são principalmente governadas por respostas comportamentais
(Figura 2). Predadores do tipo senta-e-espera devem influenciar a taxa de mortalidade das presas de modo compensatório à mortalidade natural e assim TMIIs tenderão a dominar (Beckerman et al. 1997). Predadores ativos com ampla utilização de habitat devem provocar DMIIs quando as presas também tiverem ampla utilização de habitat (Schmitz \& Suttle 2001). TMIIs devem emergir quando os predadores tiverem uma ampla utilização de habitat e as presas possuírem apenas uma curta área de vida (Schmitz 2003). Predadores ativos com limitada utilização de habitat devem causar TMIIs, não importando a utilização de habitat pelas presas (McIntosh \& Townsend 1996, Turner 1997, Peckarsky \& McIntosh 1998). Finalmente, predadores de estratégia senta-e-persegue devem causar tanto DMII quanto TMII (Schmitz \& Suttle 2001). Desta forma, a natureza e a extensão dos efeitos dos predadores nas comunidades dependerão da sinalização específica com que cada presa acessa o risco de predação.

\section{Utilização do Habitat pela Presa}

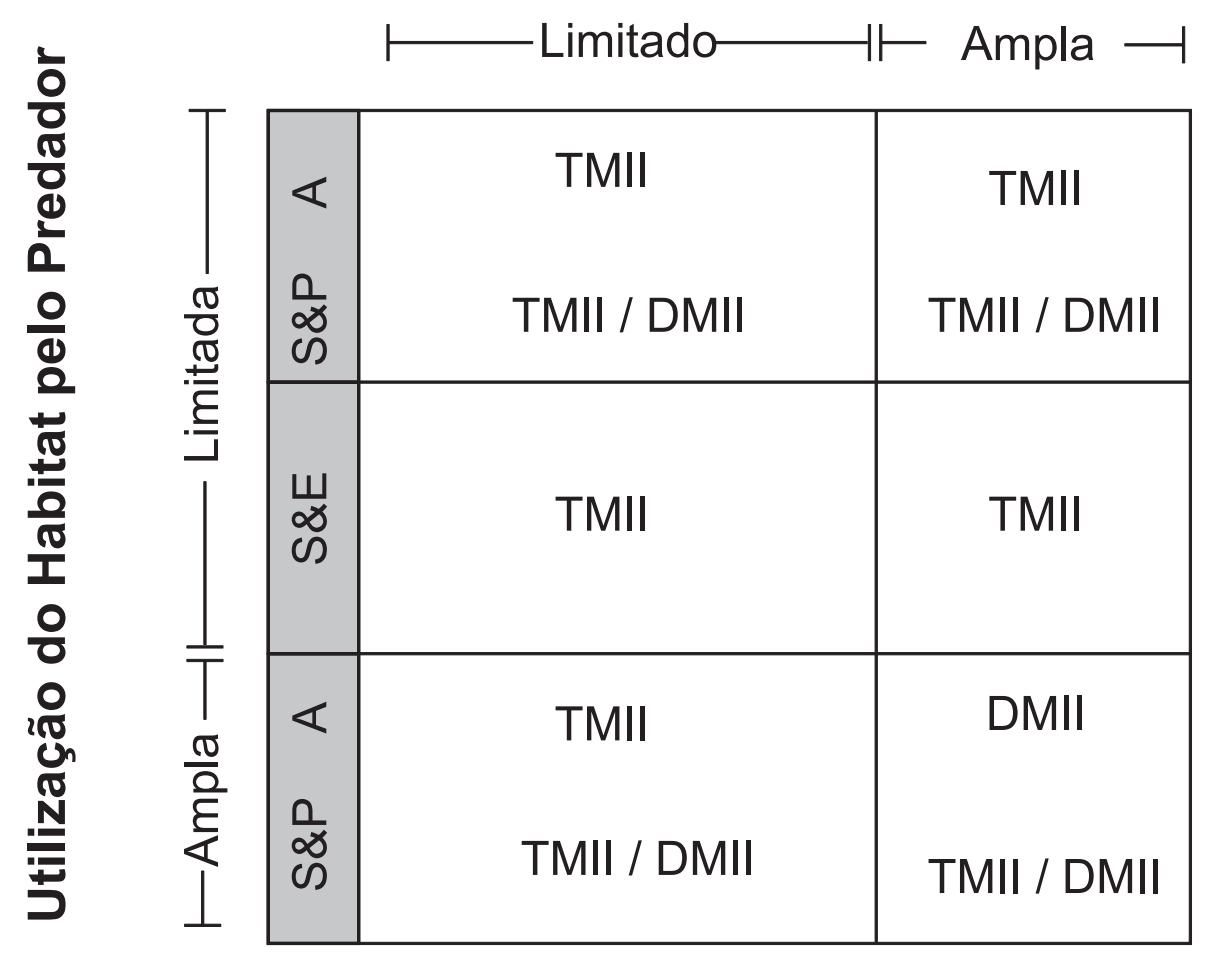

Figura 2. Tipo de interações predominantes envolvendo diferentes modos de utilização do habitat por parte das presas em função dos diferentes modos de caça e utilização do habitat dos predadores. S\&P, A e S\&E são senta-e-persegue, ativo, e senta-e-espera respectivamente.TMII representam interações indiretas mediadas por características e DMII interações indiretas mediadas por densidade. Espécies com limitada utilização do habitat selecionam apenas uma parcela de todo habitat disponível. Espécies com ampla utilização de habitat utilização toda extensão de habitat disponível. Modificado de Schmitz et al. (2004). 


\section{ABORDAGENS TEÓRICAS}

Uma série de estudos teóricos mostrou que mudanças comportamentais podem alterar as dinâmicas da comunidade em uma grande amplitude de cenários ecológicos (Bolker et al. 2003). A inserção do forrageamento adaptativo em estudos teóricos mostrou alterar a direção esperada da mudança na densidade das populações quando a comunidade é manipulada (Abrams 1992); a dinâmica das populações e a persistência de comunidades (Abrams 1982, Holt 1984, Krivan 1996, 1997, Schmitz et al. 1997, Brown et al. 1999, van Baalen et al. 2000, Attayde et al. 2006); a força relativa de interações diretas e indiretas (Abrams 1995); e o número de interações nas teias tróficas (Matsuda et al. 1994,1996). Apesar do crescente número de evidências, a importância deste tipo de interação ainda não foi absorvida pela maioria dos ecólogos de comunidades. Sejam ecólogos teóricos ou não, a maioria dos pesquisadores continuam a assumir características fixas e pareamentos independentes de interações em seus modelos e experimentos. Bolker et al. (2003) apontam três razões possíveis para explicar esta incongruência: 1 - a profusão da teoria, o que pode tornar sua assimilação difícil para não-especialistas; 2 - o foco geral da teoria e de experimentos em efeitos de curta duração, negligenciando diferentes contextos ecológicos; e 3 - a falta de conexão entre a teoria e sua base empírica.

Apesar do grande número de trabalhos sobre os efeitos comportamentais em sistemas com dois níveis tróficos (ver Bolker et al. 2003), sistemas com três níveis tróficos (predadores, consumidores e recursos) ainda não foram muito explorados teoricamente. Alguns exemplos foram proporcionados por Schwinning \& Rosenzweig (1990) e Luttbeg \& Schmitz (2000) explorando efeitos comportamentais baseados na escolha de habitat; Krivan (2000) explorando o papel da seleção alimentar, incluindo onivoria; e Abrams (1984, 1991), Fryxell \& Lundberg (1998) e Luttberg \& Schmitz (2000) avaliando as conseqüências das modificações no esforço de forrageamento na dinâmica de comunidades. No entanto, sistemas com três níveis tróficos são bastante instáveis, tornando difícil acessar tanto as densidades quanto a estabilidade relativa da comunidade em modelos com ou sem a contribuição de TMII.
Adicionalmente, devido à existência de interações não lineares (resposta funcional do tipo II, Holling 1959), sistemas com três níveis tróficos apresentam comportamento caótico para uma grande amplitude de parâmetros utilizados (McCann \& Yodziz 1994). Entretanto, existem formulações razoáveis que permitem a incorporação de TMII nestes modelos, como, por exemplo, fixar a dinâmica das populações e focar apenas as mudanças comportamentais e a distribuição das populações no espaço (Schwinning \& Rosenzweig 1990, Abrams 1991). No entanto, apesar da importância dos testes qualitativos destes modelos, investir em testes quantitativos (forma de respostas funcionais e diferenças entre respostas funcionais) ainda é necessário (Bolker et al. 2003). Abrams (1990) aponta que os modelos de resposta funcional apresentam parâmetros fixos independentes da densidade de presas (ex. modelo de resposta funcional do tipo II - Holling 1959), no entanto umas séries de características funcionais dos predadores se modificam com a disponibilidade de presas. Por exemplo, a taxa de captura das presas ou o tempo total gasto para forrageamento. Desta forma, a estimativa de parâmetros a partir destes modelos quase sempre difere daquela obtida por observações diretas. Abrams (1990) argumenta que a habilidade de se entender sistemas com múltiplas espécies a partir de seus subcomponentes não pode ser alcançada a não ser com a utilização de modelos de resposta funcional apropriados, e que para isso seja alcançado estes modelos devem incluir em sua formulação o componente de adaptação comportamental.

\section{CONSIDERAÇÕES FINAIS}

A teoria ecológica clássica assume que para entendermos a estrutura trófica dos ecossistemas é necessário apenas caracterizá-los em termos das mudanças líquidas no número ou nas densidades dos indivíduos no nível das populações, abstraindo detalhes em pequena escala como as interações no nível de indivíduos (Ovadia \& Schmtiz 2002). No entanto, os indivíduos em uma população variam quanto às suas características e respostas fenotípicas dependendo do ambiente biótico e abiótico, podendo afetar de forma significativa propriedades ecossistêmicas em grande escala (Ovadia \& Schmitz 2002). No presente trabalho, foi sintetizado um 


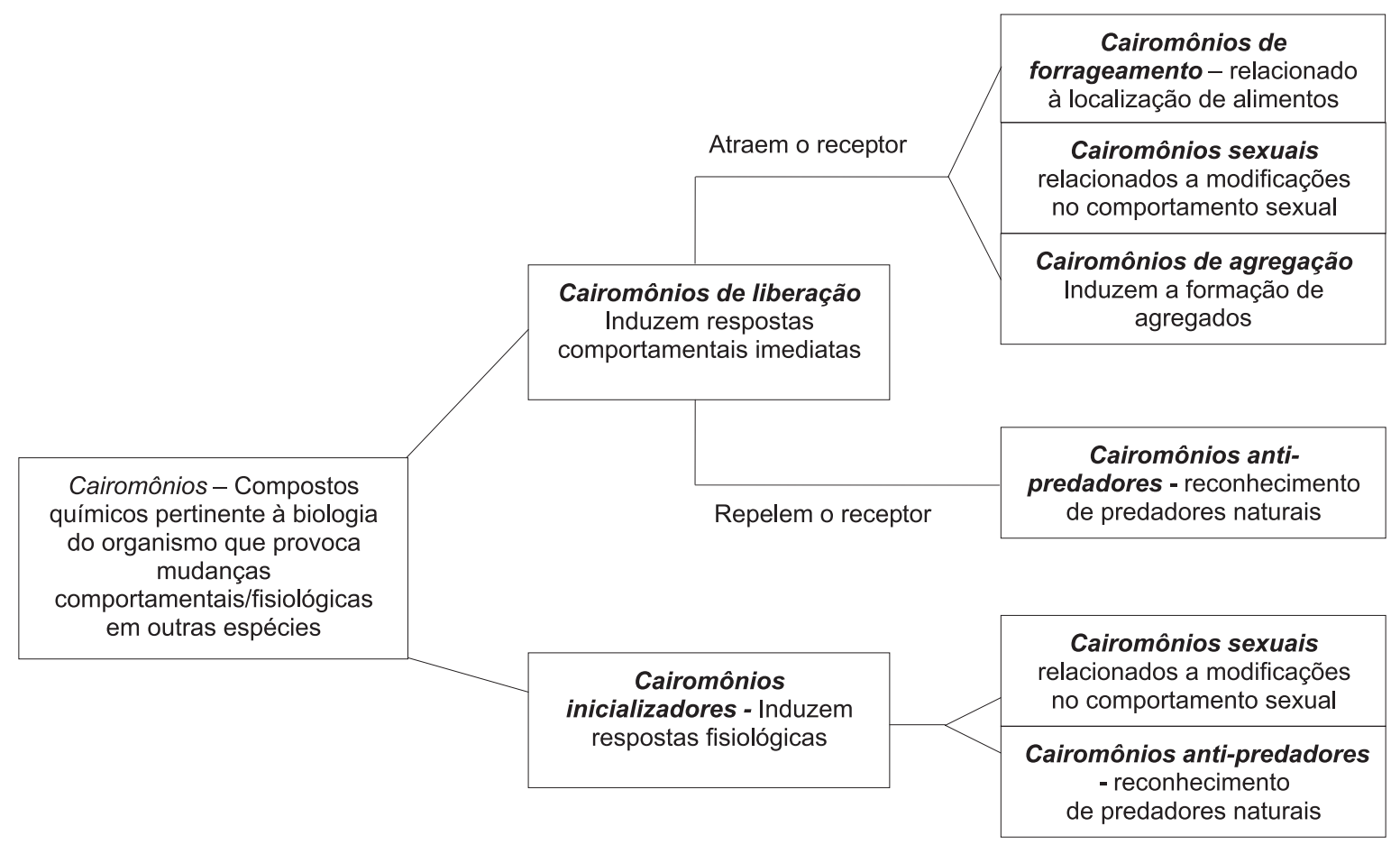

Figura 3. Classificação de cairomônios de acordo com seus efeitos. Modificado de Ruther et al. 2002.

grande número de evidências que apontam os efeitos das respostas comportamentais que atuam no nível de indivíduos (ex. trade-off entre o risco de predação e a atividade de forrageamento) na ocorrência de cascata trófica em diferentes ecossistemas. Brown et al. (1999) sugerem que os sistemas ecológicos devem ser vistos como 'paisagens de medo' onde todas as interações indiretas seriam majoritariamente mediadas por mudanças comportamentais ou outras mudanças fenotípicas. Para tanto, existem algumas lacunas que ainda necessitam serem preenchidas:

- Respostas comportamentais devem ser avaliadas em experimentos onde os predadores não são capazes de consumir suas presas e a sobrevivência das presas deve ser medida na presença e ausência de predadores.

- Estes estudos devem acessar tanto os efeitos de risco como os efeitos da predação na sobrevivência das presas, separando assim, efeitos da densidade e comportamentais (Peacor \& Werner 2001).

- A literatura fornece poucas evidências de impactos de TMIIs de longa duração e portanto experimentos devem ser conduzidos no sentido de elucidar como a força e a natureza das interações se comportam num longo período de tempo.

- Os trabalhos teóricos e empíricos sobre TMII até agora focaram diferentes aspectos da teoria (Werner \& Peacor 2003). Portanto, para que um progresso efetivo seja alcançado é necessário que as predições feitas a partir de trabalhos teóricos sejam operacionalizáveis em testes empíricos.

O ajuste do fenótipo de uma espécie em função da presença de um predador pode refletir a resposta evolutiva à variação temporal ou espacial desta interação resultando em importantes modificações na estrutura trófica de comunidades naturais (Agrawal 2001). O crescente corpo teórico e de evidências empíricas sugere que TMIIs são comuns em vários tipos de ecossistemas com importantes conseqüências na estrutura e funcionamento de teias tróficas. $\mathrm{O}$ reconhecimento de sua importância será crucial para elucidar os mecanismos que controlam as cascatas tróficas bem como o funcionamento e a estrutura de comunidades ecológicas.

AGRADECIMENTOS: Agradeço as valiosas sugestões de Jayme Santangelo, Anderson Gripp, Francisco Esteves, Reinaldo Bozelli, Vinícius Farjalla e Miriam Albrecht para a melhoria da estrutura e concepção deste trabalho. Em especial agradeço a Albert Suhett pela minuciosa e criteriosa correção textual (ortografia e sintaxe) deste trabalho. O autor é extremamente grato ao Programa de Pós Graduação em Ecologia da UFRJ, ao laboratório de Limnologia da UFRJ e ao $\mathrm{CNPq}$ por conceder a bolsa de estudos que suportou sua dissertação de mestrado. 


\section{REFERÊNCIAS}

ÅBJONRSSON K.; HANSSON L.A. \& BRONMARK C. 2004. Responses of prey from habitats with different predator regimes: Local adaptation and heritability. Ecology, 85: 1859-1866.

ABRAMS, P.A. 1984. Foraging interactions and interactions in food webs. The American Naturalist, 124: 80-96.

ABRAMS, P.A. 1990. The Effects of Adaptive Behavior on the Type-II Functional Response. Ecology, 71:877-885.

ABRAMS, P.A. 1991. Strengths of indirect effects generated by optimal foraging. Oikos, 62: 162-176.

ABRAMS, P.A. 1992. Predators that benefit prey and prey that harm predators: unusual effects of interacting foraging adaptations. The American Naturalist, 140: 573-600.

ABRAMS, P.A. 1995. Implications of dynamically variable traits for identifying, classifying, and measuring direct and indirect effects in ecological communities. The American Naturalist, 146: $112-134$.

AGRAWAL, A. 2001. Phenotypic plasticity in the interaction and evolution of species. Science, 294: 321-326.

ANHOLT, B.R. \& WERNER, E.E. 1995. Interaction between food availability and predation mortality mediated by adaptive behavior. Ecology, 76: 2230-2234.

ATTAYDE, J.L.; ISKIN, M. \& CARNEIRO, L. 2006. O papel da Onívoria na Dinâmica de Cadeias Alimentares. Oecologia brasiliensis, 10: 69-77

BECKERMAN, A.P.; URIARTE, M. \& SCHMITZ, O.J. 1997. Experimental evidence for a behavior-mediated trophic cascade in a terrestrial food chain. Proceedings of the National Academy of Sciences (USA), 94: 10735-10738.

BERNOT, R..J. \& TURNER, A.M. 2001. Predator identity and trait-mediated indirect effects in a littoral food web. Oecologia, 129: 139-146.

BOLKER, B.; HOLYOAK, M.; KRIVAN, V.; ROWE, L. \& SCHMITZ, O. 2003. Connecting theoretical and empirical studies of trait-mediated interactions. Ecology, 84: 1101-1114.

BORER, E.T.; SEABLOOM, E.W.; SHURIN, J.B.; ANDERSON, K.E.; BLANCHETTE, C.A.; BROITMAN, B.; COOPER, S.D. \& HALPERN, B.S. 2005. What determines the strength of a trophic cascade? Ecology, 86: 528-537.

BOUSKILA, A. 2001. A habitat selection game of interactions between rodents and their predators. Ann. Zoo. Fennici, 38: $55-70$.

BRÖNMARK, C. \& HANSSON, L.A. 2000. Chemical communication in aquatic systems: an introduction. Oikos, 88: 103-109

BROWN, J.S.; LAUNDRE, J.W. \& GURUNG, M. 1999. The ecology of fear: optimal foraging, game theory and trophic interactions. Journal of Mammoloy, 80: 385-399.
CARPENTER, S.R.; KITCHELL, J.F.; HODGSON, J.R.; COCHRANE, P.A.; ELSER, J.J. \& ELSER, M.M. 1987. Regulation of lake primary productivity by food web structure. Ecology, 68: 1863-1876.

DIEHL, S. \& EKLOV, P. 1995. Effects of piscivore-mediated habitat use on resources, diet and growth of perch. Ecology, 76: $1712-1726$.

DUDLEY, S.A. \& SCHMITT, J. 1996. Testing the adaptive plasticity hypothesis: density dependent selection on manipulated stem length in Impatiens capensis. The American Naturalist, 147: 445-465.

FEENER, D.H. 2000. Is the assembly of ant communities mediated by parasitoids? Oikos, 90: 79-88.

FRYXELL, J.M. \& LUNDBERG, P. 1998. Individual behavior and community dynamics. Chapman and Hall, New York, New York, USA. 202pp.

GASTREICH, K.R. 1999. Trait-mediated indirect effects of a theridid spider on an ant-plant mutualism. Ecology, 80: 1066-1070.

HASSELL, M.P. 1978. The dynamics of arthropod predator preysystems. Princeton University Press, Princeton, New Jersey. USA. 237pp.

HOLLING, C.S. 1959. The components of predation as revealed by a study of small mammal predation of the European pine sawfly. Canadian Entomologist, 91: 293-320.

HOLT, R.D. 1984. Spatial heterogeneity, indirect interactions, and the coexistence of prey species. The American Naturalist, 124: 377-406.

HUANG, C.F. \& SIH, A. 1991. Experimental studies on direct and indirect interactions in a three trophic-level stream system. Oecologia, 85: 530-536.

KATS, L. \& DILL, L.M. 1998. The scent of death: chemosensory assessment of predation risk by prey animals. Ecoscience 5: 361-394.

KRIVAN, V. 1996. Optimal foraging and predator-prey dynamics. Theoretical Population Biology, 49: 265-290.

KRIVAN, V. 1997. Dynamic ideal free distribution: effects of optimal patch choice on predator-prey dynamics. The American Naturalist, 149: 164-178.

KRIVAN, V. 2000. A mechanistic model for partial preferences. Theoretical Population Biology, 58: 279-289.

LUBCHENCO, J. 1978. Plant species diversity in a marine intertidal community: importance of herbivore food preference and algal competitive abilities. The American Naturalist, 112: 23-39.

LUDWIG, D. \& ROWE, L. 1990. Life history strategies for energy gain and predator avoidance under time constraints. The American Naturalist, 135: 696-707.

LUTTBEG, B. \& SCHMITZ, O. 2000. Predator and prey models 
with flexible individual behavior and imperfect information. The American Naturalist, 155: 669-683.

LUTTBEG B.; LOCKE, R. \& MANGEL, M. 2003. Prey state and experimental design affect relative size of Trait- and densitymediated indirect interactions. Ecology, 84: 1140-1150.

MATSUDA, H.; HORI, M. \& ABRAMS, P.A. 1994. Effects of predator-specific defense on community complexity. Evolutionary Ecology, 10: 13-28.

MATSUDA, H.; HORI, M. \& ABRAMS, P.A. 1996. Effects of predator-specific defence on biodiversity and community complexity in two-trophic level communities. Evolutionary Ecology, 10: 13-28.

McCANN, K. \& YODZIS, P. 1994. Biological conditions for chaos in a three-species food chain. Ecology, 75: 561-564.

MCINTOSH, A.R. \& TOWNSEND, C.R. 1996. Interactions between fish, grazing invertebrates and algae in a New Zealand stream: a trophic cascade mediated by fish induced changes in grazer behavior. Oecologia, 108: 174-181.

McINTOSH, A.R. \& PECKARSKY, B.L. 1999. Criteria determining behavioral responses to multiple predators by a stream mayfly. Oikos, 85: 554-564.

MESSINA, F.J. 1981. Plant protection as a consequence of an ant membracid mutualism: interactions on goldenrod (Solidago sp.). Ecology, 62: 1433-1440.

MURDOCH, W. \& OATEN, A. 1975. Predation and population stability. Advances in Ecological Research, 9: 100-131.

NYSTRÖM, P. \& ÅBJÖRNSSON, K. 2000. Effects of fish chemical cues on the interactions between tadpoles and crayfish. Oikos, 88: 181-190.

OVADIA, O. \& SCHMITZ, O.J. 2002. Linking individuals with ecosystems: identifying the dynamically relevant organizational scale for predicting trophic abundances. Proceeding of the National Academy of Science (USA), 99: 12927-12931.

PAINE, R.T. 1980. Food webs: linkage, interaction strength, and community infrastructure. Journal of Animal Ecology, 49: 667-685.

PEACOR, S.D. \& WERNER, E.E. 2000. The effects of a predator on an assemblage of consumers through induced changes in consumer foraging behavior. Ecology, 81: 1998 - 2010.

PEACOR, S.D. \& WERNER, E.E. 2001. The contribution of trait-mediated indirect effects to the net effects of a predator. Proceedings of the National Academy of Sciences (USA), 98: 3904-3908.

PECKARSKY, B.L. \& MCINTOSH, A.R. 1998. Fitness and community consequences of avoiding multiple predators. Oecologia, 113: 565-576.

ROSENZWEIGM, L. \& MACARTHUR, R.H. 1963. Graphical representation and stability of predatorprey interaction. The American Naturalist, 97: 209-223.
RUTHER J.; MEINERS T. \& STEIDLE, J.L.M. 2002. Rich in phenomena-lacking in terms. A classification of kairomones. Chemoecology, 12: 161-167.

SCHMITZ O.J.; KRIVAN, V.L. \& OVADIA, O. 2004. Trophic cascades: the primacy of trait-mediated indirect interactions. Ecology Letters, 7: 153-163.

SCHMITZ, O.J.; BECKERMAN, A.P. \& O’BRIEN, K.M. 1997. Behaviorally mediated trophic cascades: effects of predation risk on food web interactions. Ecology, 78: 1388-1399.

SCHMITZ, O.J. \& SUTTLE, K.B. 2001. Effects of top predator species on direct and indirect interactions in a food web. Ecology, 82: 2072-2081.

SCHMITZ, O.J. 2003. Top predator control of plant biodiversity and productivity in an old-field ecosystem. Ecology Letters, 6: $156-163$.

SCHWINNING, S. \& ROSENZWEIG, M.L. 1990. Periodic oscillations in an ideal-free predator-prey distribution. Oikos, 59: 85-91.

TOLLRIAN, R. \& HARVELL, C.D. 1999. The ecology and evolution of inducible defenses. Princeton University Press, Princeton, New Jersey, USA. 383pp.

TRUSSELL, G.C.; EWANCHUK, P.J. \& BERTNESS, M.D. 2002. Field evidence for trait-mediated indirect interactions in a rocky intertidal food web. Ecology Letters, 5: 241-245.

TURNER , A.M.; FETTEROLF, S.A. \& BERNOT, R.J. 1999. Predator identity and consumer behavior: differential effects of fish and crayfish on the habitat use of a freshwater snail. Oecologia, 118: 242-247.

TURNER, A.M. 1997. Contrasting short-term and long-term effects of predation risk on consumer habitat use and resources. Behavioral Ecology, 8: 120-125.

van BAALEN, M.; KRIVAN, V.; van RIJN, P.C.J. \& SABELIS, M. 2000. Alternative food, switching predators, and the persistence of predator-prey systems. The American Naturalist, 157: 512-524.

WERNER, E.E. 1992. Individual behavior and higher-order species interactions. The American Naturalist, 140: S5-S32.

WERNER, E.E. \& ANHOLT, B.R. 1993. Ecological consequences of the tradeoff between growth and mortality rates mediated by foraging activity. The American Naturalist, 142: 242-272.

WERNER, E.E. \& PEACOR, S.D. 2003. A review of traitmediated indirect interactions in ecological communities. Ecology, 84: 1083-1100.

WISSINGER, S. \& McGRADY, J. 1993. Intraguild predation and competition between larval dragonflies: direct and indirect effects of shared prey. Ecology, 74: 207-218.

Submetido em 5/12/2007 Aceito em 21/12/2007 